Revue québécoise de droit international

Quebec Journal of International Law

Revista quebequense de derecho internacional

\title{
La responsabilité civile de l'Organisation des Nations Unies. Effectivité et efficacité des mécanismes de réparation offerts pour les personnes privées : le cas des exactions sexuelles commises par les casques bleus
}

\section{Marion Mompontet}

Volume 30, numéro 1, 2017

URI : https://id.erudit.org/iderudit/1053757ar

DOI : https://doi.org/10.7202/1053757ar

Aller au sommaire du numéro

Éditeur(s)

Société québécoise de droit international

ISSN

0828-9999 (imprimé)

2561-6994 (numérique)

Découvrir la revue

Citer cet article

Mompontet, M. (2017). La responsabilité civile de l'Organisation des Nations Unies. Effectivité et efficacité des mécanismes de réparation offerts pour les personnes privées : le cas des exactions sexuelles commises par les casques bleus. Revue québécoise de droit international / Quebec Journal of International Law / Revista quebequense de derecho internacional, 30(1), 41-63.

https://doi.org/10.7202/1053757ar
Résumé de l'article

Les opérations de maintien de la paix de l'Organisation des Nations Unies (OMPs) font régulièrement la une des médias mais ce n'est pas toujours pour leurs succès. Le nombre croissant d'OMPs déployées par le Conseil de Sécurité de l'Organisation des Nations Unies a également vu augmenter le nombre d'infractions sexuelles dans les pays hôtes. Ainsi l'année 2016 a été particulièrement marquée par des allégations d'exploitation et de violences sexuelles à l'encontre de femmes et de jeunes enfants, de sexe féminin comme masculin, commises par des membres des OMPs, et tout particulièrement des Casques bleus. Selon un rapport du nouveau Secrétaire général de l'Assemblée des Nations Unies, António Guterres, paru le 28 février 2017, rien que pour l'année 2016, 65 allégations d'exploitation et violences sexuelles ont été proférées à l'encontre du personnel civil, contre 80 allégations pour le personnel en uniforme. Au total, 311 victimes étaient concernées. Si beaucoup d'encre a déjà coulé sur le sujet de la responsabilité pénale individuelle des Casques bleus et le cadre juridique de leur action, peu a encore été dit sur la part de responsabilité que l'ONU détient dans la commission de ces graves infractions sexuelles. L'ONU accepte-t-elle sa part de responsabilité ? Indemnise-t-elle les victimes ? Cet article vise donc à analyser les mécanismes qu'offre l'Organisation des Nations Unies pour recenser les plaintes et indemniser les victimes d'exploitation et de violences sexuelles par les membres des opérations de maintien de la paix. Deux problèmes importants apparaissent alors : si bien des mécanismes sont élaborés, leur absence d'effectivité et d'efficacité et le refus flagrant de l'ONU d'accepter sa responsabilité civile en matière d'exploitation et de violences sexuelles subsistent à travers les années.
Tous droits réservés @ Société québécoise de droit international, 2018
Ce document est protégé par la loi sur le droit d'auteur. L’utilisation des services d'Érudit (y compris la reproduction) est assujettie à sa politique d'utilisation que vous pouvez consulter en ligne.

https://apropos.erudit.org/fr/usagers/politique-dutilisation/ 


\title{
LA RESPONSABILITÉ CIVILE DE L'ORGANISATION DES NATIONS UNIES. EFFECTIVITÉ ET EFFICACITÉ DES MÉCANISMES DE RÉPARATION OFFERTS POUR LES PERSONNES PRIVÉES : LE CAS DES EXACTIONS SEXUELLES COMMISES PAR LES CASQUES BLEUS
}

\author{
Marion Mompontet ${ }^{*}$
}

\begin{abstract}
Les opérations de maintien de la paix de l'Organisation des Nations Unies (OMPs) font régulièrement la une des médias mais ce n'est pas toujours pour leurs succès. Le nombre croissant d'OMPs déployées par le Conseil de Sécurité de l'Organisation des Nations Unies a également vu augmenter le nombre d'infractions sexuelles dans les pays hôtes. Ainsi l'année 2016 a été particulièrement marquée par des allégations d'exploitation et de violences sexuelles à l'encontre de femmes et de jeunes enfants, de sexe féminin comme masculin, commises par des membres des OMPs, et tout particulièrement des Casques bleus. Selon un rapport du nouveau Secrétaire général de l'Assemblée des Nations Unies, António Guterres, paru le 28 février 2017, rien que pour l'année 2016, 65 allégations d'exploitation et violences sexuelles ont été proférées à l'encontre du personnel civil, contre 80 allégations pour le personnel en uniforme. Au total, 311 victimes étaient concernées. Si beaucoup d'encre a déjà coulé sur le sujet de la responsabilité pénale individuelle des Casques bleus et le cadre juridique de leur action, peu a encore été dit sur la part de responsabilité que l'ONU détient dans la commission de ces graves infractions sexuelles. L'ONU accepte-telle sa part de responsabilité ? Indemnise-t-elle les victimes? Cet article vise donc à analyser les mécanismes qu'offre l'Organisation des Nations Unies pour recenser les plaintes et indemniser les victimes d'exploitation et de violences sexuelles par les membres des opérations de maintien de la paix. Deux problèmes importants apparaissent alors : si bien des mécanismes sont élaborés, leur absence d'effectivité et d'efficacité et le refus flagrant de l'ONU d'accepter sa responsabilité civile en matière d'exploitation et de violences sexuelles subsistent à travers les années.
\end{abstract}

The United Nations Organisation's peacekeeping operations are regularly cited in the medias but it is not always for their successes. The growing number of peacekeeping operations deployed by the Security Council of the United Nations has also seen the number of sexual exploitation and abuse increase in the host countries. For instance, the year 2016 has been strongly marked by allegations of sexual exploitation and abuse against women and children, girls as boys, perpetrated by members of peacekeeping operations, especially blue helmets. Among a report of the new Secretary general of the UN general assembly, António Guterres, published $28^{\text {th }}$ February 2017, only for the year 2016, 65 allegations of sexual exploitation and abuse has been attributed to the civilan staff and 80 attributed to the uniformed personnel for a total of 311 victims. If a lot of things has been already written about the individual criminal responsibility of the blue helmets and the legal framework of their action, very little has been said about the United Nation's share of responsibility in the commission of this serious sexual offences. Does the UNO accept its share of responsibility? Does it indemnify the victims? This article aims to analyse the different mechanisms offered by the United Nations to register complaints and indemnify the victims of sexual exploitation and abuse committed by the members of the peacekeeping operations. Two major problems appear about that question: if lots of mechanisms are created, the absence of effectivity and effectiveness and the obvious refusal of the UNO in accepting its civil responsibility as regards sexual exploitation and abuse remain through the years.

Las operaciones de mantenimiento de la paz de las Naciones unidas aparecen regularmente en los periódicos, pero no siempre es para sus éxitos. El creciente número de operaciones de mantenimiento de la paz desplegadas por el Consejo de Seguridad de las Naciones unidas también ha visto el número de delitos sexuales en los países de acogida aumentar. Así, el año 2016 fue particularmente marcado por acusaciones de explotación y de violencias sexuales contra las mujeres y los niños, niñas como niños, cometidas por los

* Candidate à la maitrise en droit international, Université du Québec à Montréal. 
miembros del mantenimiento de la paz, especialmente cascos azules. Según un informe del nuevo Secretario general de la Asamblea de las Naciones unidas, António Guterres, publicado el 28 de febrero de 2017, sólo para el año 2016, 65 denuncias de explotación y abusos sexuales se han hecho contra el personal civil y 80 denuncias contra el personal en uniforme para un total de 311 víctimas. Si numerosos artículos fueron escribidos sobre el tema de la responsabilidad individual y penal de los cascos azules y el marco jurídico de sus acción, poco se ha dicho a propósito de la responsabilidad que las Naciones Unidas posee en la comisión de estos serios delitos sexuales. ¿Las Naciones unidas aceptan su parte de responsabilidad? ¿Se compensa a las víctimas? Este artículo tiene como objetivo de analizar los mecanismos ofrecidos por las Naciones unidas para identificar las denuncias y reparar a las víctimas de explotación y violencias sexuales por parte de miembros de las operaciones del mantenimiento de la paz. Dos cuestiones importantes aparecen a continuación: así se desarrollan mecanismos, su falta de eficacia y eficiencia, y el flagrante rechazo de las Naciones unidas para aceptar las cuestiones operativas de responsabilidad civil y la violencia sexual persisten a través de los años. 
L'ONU devrait faire preuve de leadership pour mettre un terme à l'exploitation et aux abus sexuels partout dans le système, du siège à New York aux missions sur le terrain dans les zones de conflit. Les gouvernements devraient arrêter de faire bonne figure devant ce problème pour prendre des actions concrètes comme améliorer la formation de leurs troupes et sanctionner les responsables d'abus.

Sarah Taylor, responsable de plaidoyer auprès de la division Droits des femmes à Human Rights Watch ${ }^{1}$.

Le succès et la légitimité des opérations de maintien de la paix (OMPs) de l'Organisation des Nations Unies (ONU) ont été plusieurs fois ternis par les crimes commis par son personnel. En effet, en 2016 $6^{2}$, plusieurs scandales ont éclaté concernant différentes infractions sexuelles commises à l'encontre de femmes et d'enfants, allant du viol à l'agression sexuelle, en passant par la prostitution forcée. Si plusieurs OMPs sont concernées par ces graves violations du droit international humanitaire et des droits de l'homme, comme la Mission des Nations Unies en Sierra Leone (MINUSIL) ou la Mission de l'Organisation des Nations Unies pour la stabilisation en République Démocratique du Congo (MONUSCO) pour ne citer que celles-ci ${ }^{3}$, l'opération la plus touchée est sans conteste la Mission des Nations Unies en République centrafricaine (MINURCA) qui est en cours ${ }^{4}$. En avril 2016, elle a encore défrayé la chronique lorsque des allégations de viols et d'agressions sexuelles particulièrement horribles commises par des Casques bleus et des soldats de l'opération française Sangaris sont apparues dans la presse ${ }^{5}$. Or, les infractions sexuelles sont réprimées en droit national ${ }^{6}$, en droit international ${ }^{7}$, ainsi qu'en droit

1 Human Rights Watch, communiqué, «ONU : mettre fin aux abus sexuels commis par des Casques bleus » (4 mars 2016), en ligne : <www.hrw.org>.

2 Selon un rapport du Secrétaire général des Nations Unies, en 2016, 65 allégations d'exploitation et d'atteintes sexuelles ont été formulées à l'encontre du personnel civil onusien et 80 à l'encontre du personnel en uniforme concernant un total de 311 victimes. Voir António Guterres, Dispositions spéciales visant à prévenir l'exploitation et les atteintes sexuelles : une nouvelle stratégie, Doc off AG NU, 71 ${ }^{\mathrm{e}}$ sess, Doc NU A/71/818 (2017) 1 à la p 5 [Dispositions spéciales].

3 Céline Boff, «1992-2016 : Les Casques bleus régulièrement accusés de crimes sexuels », 20minutes.fr ( $1^{\mathrm{er}}$ avril 2016), en ligne : <www.20minutes.fr>. Voir Étude d'ensemble de toute la question des opérations de maintien de la paix sous tous leurs aspects, Doc off AG NU, 59ª sess, Doc NU A/59/710 (2005).

4 «Viols d'enfants en Centrafrique : l'"échec flagrant" de l'ONU dénoncé », La Presse (17 décembre 2015), en ligne : <www.lapresse.ca/international/afrique/201512/17/01-4932217-viols-denfants-encentrafrique-lechec-flagrant-de-lonu-denonce.php>.

5 «Soupçons d'abus sexuels en Centrafrique : Si c'est avéré, "l’honneur de la France serait engagé", affirme Hollande », 20minutes.fr ( ${ }^{\text {er }}$ avril 2016), en ligne : <www.20minutes.fr/monde/181749120160401-soupcons-abus-sexuels-centrafrique-si-avere-honneur-france-engage-affirme-hollande $>$.

6 Voir notamment art 222-22 C pén; Code criminel, LRC 1985, c C-46, art 151; 18 USC § 2242 (2017); Congreso de Colombia, 24 juillet 2000, Código penal Colombiano, Diario Oficial 44097 del 24 de julio de 2000, art 206 (Colombie); Ministre de la Justice, 5 mars 1881, Code criminel, art 242 (Danemark); Sexual Offenses Act (R-U), 2003; 27 mai 1852, Code pénal autrichien, art 201 (Autriche); $1^{\mathrm{er}}$ janvier 1872, Code criminel allemand, Federal Law Gazette, art 177 (Allemagne) [Code criminel 
international coutumier ${ }^{8}$. Ces atteintes à l'intégrité physique des personnes ont une qualification pénale propre en droit national ${ }^{9}$ et sont également des violations des droits de l'homme. Néanmoins, en droit international, ce sont des crimes sous-jacents à d'autres crimes internationaux, comme par exemple, le crime contre l'humanité en droit pénal international ${ }^{10}$ et leur qualification peut varier selon le contexte, l'époque, la juridiction et les fondements utilisés ${ }^{11}$.

allemand]; The penal code Act, National Assembly of Zambia, art 132 (Zambie) [The penal code Act]; Criminal Law of South Africa (Sexual offences and related matters) Amendment Act 2007, no 32 de 2007, art 3 [Criminal Law of South Africa]; Ministerio de Justicia y de derechos humanos, 21 décembre 1984, Código penal Argentino, art 119 (Argentine).

$7 \quad$ Statut de Rome de la Cour pénale internationale, 17 juillet 1998, 2187 RTNU 3, art 7(1)g), 8(2)b)(xxi) (xxii) (entrée en vigueur : $1^{\mathrm{er}}$ juillet 2002) [Statut de Rome]. La jurisprudence internationale, notamment par le biais des tribunaux ad hoc, a condamné ce genre d'infraction à maintes reprises. Voir Le Procureur c Anto Furundzija, IT-95-17/1-T, Jugement (10 décembre 1998) au para 172 (Tribunal pénal international pour l'ex-Yougoslavie, Chambre de première instance) [Jugement Furundzija]; Le Procureur c Jean-Paul Akayesu, ICTR-96-4-T, Jugement (2 septembre 1998) aux paras 731-32 (Tribunal pénal international pour le Rwanda, Chambre de première instance) [Jugement Akayesu]; Le Procureur c Alfred Musema, ICTR-96-13, Jugement et sentence (27 janvier 2000) au para 933 (Tribunal pénal international pour le Rwanda, Chambre de première instance) [Jugement Musema]. Ces tribunaux ouvriront la voie à la Cour pénale internationale dont l'arrêt le plus récent en matière de viol concerne le conflit en République centrafricaine. Voir Le Procureur c Jean-Pierre Bemba Gombo, ICC-01/05-01/08-2162, Jugement (9 mars 2012).

8 Jean-Marie Henckaerts et Louise Doswald-Beck, Droit international coutumier, Bruxelles, Bruylant, 2006 aux pp 427-428 [Henckaerts et Doswald-Beck].

9 Par exemple, s'agissant de la notion de viol, le droit français définit clairement le viol à l'article 222-23 du Code pénal comme étant « [t]out acte de pénétration sexuelle, de quelque nature qu'il soit, commis sur la personne d'autrui par violence, contrainte, menace ou surprise (...) ». À l'inverse d'autres législations précisent qu'un viol doit être commis à l'encontre d'un corps féminin. Voir Code criminel allemand, supra note 6, art 177; Criminal law of the people's Republic of China, 1997, art 236 (République populaire de Chine); The penal code Act, supra note 6, art 132; Japanese penal code, 1907, art 177 (Japon). Ensuite, si plusieurs législations internes précisent que l'élément matériel du viol consiste en une pénétration, fut-elle légère (voir à cet effet Criminal Law of South Africa, supra note 6; Pakistan penal code (1860), art 375 (Pakistan)) de l'organe sexuel féminin par l'organe sexuel masculin, d'autres laissent une interprétation plus large (voir New South Wales Crimes Act (1900), art 61 (Nouvelle-Galles du Sud)). Enfin, de grandes divergences existent s'agissant de la qualification de la pénétration orale forcée qui est tantôt codifiée comme une violence sexuelle, tantôt comme un viol (voir 2011, Código penal Chileno, art 361 (Chili); art 222-23 C pén.). Voir Jugement Furundzija, supra note 7 aux para 183-89.

10 Statut de Rome, supra note 7, art 7.

11 Le droit international des droits de l'homme sanctionne les violences sexuelles au travers du droit à l'intégrité physique. Voir Déclaration universelle des droits de l'homme, Rés AG 217A (III), Doc off AG NU, $3^{\mathrm{e}}$ sess, supp $\mathrm{n}^{\circ} 13$, Doc NU A/810 (1948) 71 art 5 [Déclaration universelle]; Convention de sauvegarde des droits de l'Homme et des libertés fondamentales, 4 novembre 1950, 213 RTNU 221 à l'art 3 (entrée en vigueur : 3 septembre 1953) [Convention européenne des droits de l'homme]; Pacte international relatif aux droits civils et politiques, 19 décembre 1966, 999 RTNU 171 art 7 (entrée en vigueur : 23 mars 1976) [PIDCP]. En droit international humanitaire, les différents textes, tout comme la jurisprudence, ne font pas état d'un crime à part entière mais plutôt d'un acte de torture. Voir Jugement Furundzija, supra note 7 au para 163, tantôt comme une violation des lois et coutumes de la guerre (voir Jugement Furundzija, supra note 7 au para 172) ou encore comme un élément constitutif de génocide (voir Jugement Akayesu, supra note 7 aux para 731-732; Jugement Musema, supra note 7 au para 933); voir également Statut de Rome, supra note 7, art 7, g), art 8, al. 2, b) xxi), xxii), art 8, al. 2, e) vi). 
On s'interroge alors sur les sanctions dont peuvent faire l'objet les Casques bleus (qui sont par ailleurs soumis à un régime de responsabilité extrêmement complexe puisque la responsabilité de leurs actes est partagée entre les juridictions nationales et $\mathrm{l}^{\prime} \mathrm{ONU}{ }^{12}$ ) et sur la part de responsabilité de l'ONU dans ces affaires d'exactions sexuelles. En effet, lorsque les Casques bleus participent à des OMPs prévues par des résolutions du Conseil de Sécurité ${ }^{13}$, ils n'appartiennent pas à un corps militaire onusien puisqu'il n'en existe pas. Ils font partie de contingents fournis volontairement par leur État de nationalité ${ }^{14}$. Or, en vertu de l'accord-cadre entre les Nations Unies et les pays fournisseurs de contingents, c'est au pays d'envoi que revient la compétence de poursuivre judiciairement les soldats accusés d'exactions ${ }^{15}$. Toutefois, l'ONU garde une responsabilité de commandement dans le déroulement de la mission ${ }^{16}$. Elle est donc responsable des actes commis par les membres du personnel de maintien de la paix, dans l'exercice de leurs fonctions et qui pourraient causer des dommages à des tiers ${ }^{17}$. À ce titre, l'ONU peut renvoyer des contingents entiers dans leur État d'origine et leur interdire de participer à de prochaines missions ${ }^{18}$, mais elle ne dispose pas d'un privilège de juridiction. La compétence qui lui échoit reste donc l'indemnisation des victimes sous le régime de la responsabilité civile, la responsabilité pénale individuelle relevant du droit national des États fournisseurs de contingents. Il existe donc deux niveaux de responsabilités partagés entre l'État fournisseur et l'ONU ${ }^{19}$.

Ainsi, pour Lewis Mudge, chercheur auprès de la division Afrique de l'organisation non gouvernementale Human Rights Watch, " il ne faudrait pas se contenter de renvoyer chez eux, sans engagement à l'égard de la justice, les Casques bleus qui violent, qui exploitent ou qui tuent. L'ONU devrait tirer parti de toute l'influence qu'elle exerce sur les pays qui fournissent des contingents afin de s'assurer que ceux qui soumettent des victimes à des abus et ternissent l'image de

12 Si la responsabilité pénale individuelle des Casques bleus relève des États d'envoi, la responsabilité pour une faute commise dans l'exercice de leurs fonctions relève de l'ONU. Voir Svetlana Zasova, $L e$ cadre juridique de l'action des casques bleus, Paris, Publications de la Sorbonne, 2014.

13 Les OMPs ne sont pas expressément prévues par la Charte des Nations Unies mais sont créées en vertu des chapitres 6, 7 ou 8 qui confèrent au Conseil de Sécurité la possibilité de prendre les mesures nécessaires et adéquates pour maintenir la paix et la sécurité internationales.

14 Charte des Nations Unies, 26 juin 1945 RT Can 1945 n $^{\circ} 7$ à l'art 43 [Charte des NU].

15 Demande de réformation du jugement $n^{\circ} 273$ du Tribunal administratif des Nations Unies, Avis consultatif, [1982] CIJ rec 325 à la p 360.

16 Commission du droit international, Projet d'articles sur la responsabilité des organisations internationales et commentaires y relatifs, Doc off $\mathrm{AG} \mathrm{NU}, 66^{\mathrm{e}}$ sess, supp $\mathrm{n}^{\circ} 10$, Doc NU A/66/10 (2011) art 7 [Projet d'articles]. Voir aussi Marten Zwanenburg, «UN Peace Operations between Independence and Accountability » (2008) 5 International Organizations Law Review 23 aux pp 30-31 [Zwanenburg]; International institute of humanitarian law, communiqué, « International humanitarian law, human rights and peace operations » (4-6 septembre 2008), aux pp 117-18, en ligne : International Committee of the Red Cross <www.icrc.org/eng/assets/files/other/sanremo-2008_peace_ops.pdf $>$.

17 Demandes d'indemnisation au titre de la responsabilité civile : limitations temporelles et financières, Rés AG 52/247, Doc off AG NU, 52 ${ }^{\mathrm{e}}$ sess, supp n 49, Doc NU A/Res/52/247 (1998) 1 au para 5 [Demandes d'indemnisation].

18 Opérations de maintien de la paix des Nations Unies, Rés CS 2272, Doc off CS NU, 2016, Doc NU S/RES/2272 (2016).

19 Jean-Marc Sorel, «La responsabilité des Nations Unies dans les opérations de maintien de la Paix » (2001) 3:2 Forum du droit international 127 à la p 133[Sorel]. 
l'ONU, ainsi que sa mission, répondent de leurs crimes devant les tribunaux $»^{20}$. Au soutien de cette déclaration, Human Rights Watch ajoute «qu'assurer la sécurité et fournir des services aux victimes de sévices devrait être au centre de la réponse apportée par l'ONU et le gouvernement aux abus commis par les soldats de la paix et d'autres personnels de l'ONU $»^{21}$. L'ancien Secrétaire général de l'ONU, Ban Kimoon, a d'ailleurs publié un rapport ${ }^{22}$ sur les allégations de violences et crimes sexuels commis par les Casques bleus en République centrafricaine. Il y établit des dispositions visant à prévenir l'exploitation et les atteintes sexuelles et rappelle l'importance de la promotion de la responsabilité pénale, dont la poursuite revient à l'État d'envoi ou encore à l'État hôte de l'opération ${ }^{23}$, ainsi que la mise en place de mesures correctives pour faire face à ces exactions ${ }^{24}$. Pourtant, très peu de victimes ont bénéficié d'une assistance en raison du manque de fonds dédiés à ces mécanismes et de la lenteur du processus d'application ${ }^{25}$. En effet, selon le Bureau des services de contrôle interne, seulement 26 victimes d'exploitation et d'abus sexuels sur 217 ont pu bénéficier d'une assistance, sans parler du fait qu'un flou demeure dans la signification du terme «assistance» qu'aucun document ne définit ${ }^{26}$. Ainsi, même pour les personnes ayant bénéficié d'une assistance, celle-ci reste impossible à déterminer clairement ${ }^{27}$. Cela s'explique notamment par un manque de fonds, car au moment d'écrire ces lignes, le budget moyen de 7,8 milliards de dollars alloué aux OMPs pour l'année 2016-2017 $7^{28}$ ne prévoyait pas l'indemnisation des victimes lésées par un membre de ces opérations ${ }^{29}$.

Si de nombreux articles ont déjà traité du fléau des exactions sexuelles commises par les membres des OMPs et de la responsabilité pénale des auteurs présumés ${ }^{30}$, peu d'articles se sont attardés à la responsabilité de l'ONU dans ces

20 «République centrafricaine: Des viols commis par des Casques bleus » (4 février 2016), Human Rights Watch, en ligne : <www.hrw.org/fr/news/2016/02/04/republique-centrafricaine-des-violscommis-par-des-casques-bleus $>$.

21 «ONU : Mettre fin aux abus sexuels commis par des Casques bleus » (4 mars 2016), Human Rights Watch, en ligne : <www.hrw.org/fr/news/2016/03/04/onu-mettre-fin-aux-abus-sexuels-commis-pardes-casques-bleus $>$.

22 Ban Ki-Moon, Dispositions spéciales visant à prévenir l'exploitation et les atteintes sexuelles : une nouvelle stratégie, Doc off AG NU, 70e sess, Doc NU A/70/729 (2016) [Rapport Ban].

23 Ibid au para 64.

24 Ibid aux paras 75-80.

25 Evaluation of the Enforcement and Remedial Assistance Efforts for Sexual Exploitation and Abuse by the United Nations and Related Personnel in Peacekeeping Operations, Doc off Department of Field Support NU, 2015, Doc NU IED-15-001 aux pp 23-26 [Evaluation of the Enforcement].

26 Ibid à la p 24.

27 Ibid à la $\mathrm{p} 25$.

28 ONU, Opérations de maintien de la paix, «Financer les opérations », en ligne : $<$ www.un.org/fr/peacekeeping/operations/financing.shtml $>$.

29 Réseau de recherche sur les opérations de paix, Université de Montréal, « Budget des opérations de maintien de la paix » (janvier 2013), en ligne: <www.operationspaix.net/17-resources/detailslexique/budget-des-operations-de-maintien-de-la-paix.html $>$.

30 Voir Marco Odello, «Tackling Criminal Acts in Peacekeeping Operations: the Accountability of Peacekeepers » (2010) 15:2 J Confl \& Sec L 347 [Odello]; Catherine E. Sweetser, "Providing Effective Remedies to Victims of Abuse by Peacekeeping Personnel » (2008) 83:5 NYUL Rev 1643; Richard J. Wilson et Emily Singer Hurvitz, « Human Rights Violations by Peacekeeping Forces in Somali » (2014) 21:2 Human Rights Brief 2. Dans son article, Marco Odello cite de nombreux articles 
affaires et sur la façon dont elle s'y prend pour y faire face. Il importe alors de savoir quelles sont l'efficacité et l'effectivité des mécanismes d'indemnisation mis en place par l'ONU pour la répression et la réparation des préjudices subis par les victimes d'exactions sexuelles. Il sera principalement question ici de la responsabilité civile de l'organisation et des mécanismes de réparation et non de la responsabilité individuelle des différents membres du personnel des OMPs.

$\mathrm{Si}$, de prime abord, des recherches ont permis de constater que l'ONU propose effectivement quelques solutions, comme par exemple la possibilité de recourir à un règlement à l'amiable ${ }^{31}$, aux commissions permanentes des réclamations normalement prévues par les modèles d'accord sur le statut des forces ${ }^{32}$, ou encore aux comités consultatifs internes ${ }^{33}$, notre hypothèse est que les mécanismes offerts ne sont que très peu mis en pratique et manquent grandement d'efficacité en raison, notamment, de leur complexité de mise en œuvre et de leur manque de proximité avec les victimes.

Dans un souci de clarté, il convient de préciser que l'ONU propose parfois des règlements à l'amiable faisant intervenir son Bureau des affaires juridiques ${ }^{34}$. La question portant sur l'effectivité et l'efficacité des mécanismes officiels règlementés par des textes, comme le règlement à l'amiable, même s'il y sera parfois fait référence, ne sera pas étudié en substance dans cette étude. De même, la distinction entre les différents acteurs présents dans une OMP comme les militaires, les fonctionnaires, les civils et les bénévoles ${ }^{35}$ ne sera pas analysée.

À travers une approche positiviste pragmatique puis, s'inscrivant dans la lignée des analyses offertes par Jean-Marc Sorel ${ }^{36}$ et Marten Zwanenburg ${ }^{37}$, nous analyserons les fondements de l'obligation de réparer le préjudice subi et comment ils s'appliquent à l'ONU pour indemniser les victimes d'exactions sexuelles tout en étudiant la pratique de l'organisation en matière d'indemnisation (I). Ensuite, nous analyserons leur efficacité et leur effectivité qui se confrontent à la mince frontière entre les immunités de l'ONU et l'impunité, mais également au manque de proximité avec les victimes des exactions sexuelles commises par le personnel du maintien de la paix (II).

sur le sujet. Voir Mark Pallis, "The Operation of UNHCR's Accountability Mechanisms » (2005) 37 NYUJ Intl L \& Pol 869; Susan A. Notar, "Peacekeepers as Perpetrators: Sexual Exploitation and Abuse of Women and Children in the Democratic Republic of the Congo » (2006) 14:2 J Gender Social Policy L 413.

31 Convention sur les privilèges et immunités des Nations Unies, 13 février 1946, 1 RTNU 1 art 8 (entrée en vigueur : 17 septembre 1946) [Convention sur les privilèges].

32 Modèle d'accord sur le statut des forces pour les opérations du maintien de la paix, Doc off AG NU, 1990, Doc NU A/45/594 6 au para 51 [Modèle d'accord].

33 Thomas G. Bode, «Cholera In Haiti : United Nations Immunity And Accountability » (2016) 47 Geo J Intl L 759 à la 771 [Bode]; Sorel, supra note 19 à la p 134.

34 ONU, Bureau des affaires juridiques, «Division des questions juridiques générales », en ligne : $<$ legal.un.org/ola/FR/div_gld.aspx?section=gld $>$.

35 Odello, supra note 30 à la p 365.

36 Hervé Ascensio, «Les différends opposant une organisation internationale à des personnes privées » dans Evelyne Lagrange et Jean-Marc Sorel, dir, Droit des organisations internationales, LGDJ Lextenso Éditions, Paris, 2013, 1121 [Ascensio]; Sorel, supra note 19.

37 Zwanenburg, supra note 16. 


\section{La méconnaissance des fondements de l'obligation de réparer via l'usage des mécanismes officieux au détriment des mécanismes de plaintes spécialement mis en place par l'ONU}

Nous verrons ici que bien que l'ONU ne soit partie à aucun traité sur les droits de l'homme ${ }^{38}$, elle est néanmoins soumise à la protection et à la promotion de ces droits ${ }^{39}$. Pour cette raison, elle est également tenue de réparer le préjudice causé par ses agents. Il convient donc dans un premier temps de déterminer les fondements de l'obligation de réparer et de s'attarder sur les textes qui fondent l'interdiction des violences sexuelles (A) puis de voir comment l'ONU met ces principes en pratique (B).

\section{A. L’obligation de protéger les civils et de réparer le préjudice subi}

L'Assemblée générale de l'ONU prévoit, au travers d'une résolution, l'obligation d'assurer aux victimes des violations des droits de l'homme et du droit international humanitaire un accès effectif à la justice et à la réparation du préjudice qu'elles ont $\operatorname{subi}^{40}$. Nous pouvons en déduire que si les États sont soumis à cette obligation, l'ONU l'est également ${ }^{41}$; elle possède donc, elle aussi, une obligation de réparer qui repose cependant sur des fondements différents. En effet, dans le cadre des OMPs, même si ce sont les États qui fournissent les contingents militaires et qui bénéficient donc du privilège de juridiction, l'ONU détient également une part de responsabilitét $^{42}$ en ce que les OMPs sont des organes subsidiaires du Conseil de Sécurité $^{43}$. À ce titre, l'organisation a, entre autres, le devoir de s'assurer de la formation adéquate du personnel envoyé sur le terrain ainsi que du bon déroulement de la mission et surtout de la protection des civils ${ }^{44}$.

Les textes restent évasifs sur les modes de réparation précis que l'ONU devrait mettre en place. Toutefois, un élément de réponse apparaît dans la Convention sur les privilèges et immunités des Nations Unies $^{45}$. En effet, si l'ONU n'a pas

38 Odello, supra note 30 à la p 364.

39 Charte des NU, supra note 14, arts 1(3), 55(c), 56.

40 Principes fondamentaux et directives concernant le droit à un recours et à réparation des victimes de violations flagrantes du droit international des droits de l'homme et de violations graves du droit international humanitaire, Rés AG 60/147, Doc off AG NU, 60 sess, Doc NU A/RES/60/147 (2005) [Principes fondamentaux et directives].

41 L'ONU doit pratiquer ce qu'elle prêche, sans quoi elle n'aurait pas grande crédibilité sur la scène internationale. Voir Pierre Bodeau-Livinec, « La réforme de l'administration de la justice aux Nations Unies » (2008) 54:1 AFDI 305 à la p 306 [Bodeau-Livinec].

42 Stratégie globale visant à éliminer l'exploitation et les abus sexuels dans les opérations de maintien de la paix des Nations Unies, Rés AG 59/710, Doc off AG NU, 59ess, Doc NU A/59/710 (2005) au para 38 [Stratégie globale].

43 Conseil de Sécurité des Nations Unies, «Organes subsidiaires du Conseil de Sécurité », en ligne : $<$ www.un.org/sc/suborg/fr>.

44 Ibid.

45 Convention sur les privilèges, supra note 31. 
d'obligation de prévoir des mécanismes juridictionnels, elle doit toutefois prévoir une alternative; cela peut donc être des modes de règlements alternatifs ${ }^{46}$. L'absence d'organisme juridictionnel compétent pour régler les litiges entre les personnes privées et l'ONU n'empêche pas cette dernière d'assumer la responsabilité pour ses violations au droit international ${ }^{47}$. C'est la raison pour laquelle l'ONU se doit d'indemniser les victimes d'infractions commises par le personnel qu'elle envoie, quand bien même ces infractions seraient commises en dehors de leurs fonctions, comme les exactions sexuelles par exemple.

Si l'on se penche du côté du droit international humanitaire (DIH), on constate que les forces de maintien de la paix peuvent y être soumises lorsqu'elles sont considérées comme des parties au conflit armé, dépendamment du mandat sous lequel les OMPs ont été créées. Néanmoins, l'ONU ne peut pas devenir partie aux traités fondateurs du $\mathrm{DIH}^{48}$. Il n'en demeure pas moins que, même si l'organisation n'est pas elle-même soumise aux obligations du DIH conventionnel, les États contributeurs de troupes qui ont ratifié les Conventions de Genève y sont quant à eux soumis $^{49}$ lorsqu'il a vocation à s'appliquer.

En revanche, il convient de mentionner que lorsque les critères nécessaires à l'application du DIH sont réunis ${ }^{50}$, l'ONU est soumise au respect du DIH coutumier ${ }^{51}$. Or, les violences sexuelles sont prohibées tant par le droit conventionnel ${ }^{52}$ que par le droit coutumier ${ }^{53}$. Donc en toute logique, lorsqu'ils sont commis par des Casques bleus, ces crimes seraient susceptibles de faire l'objet de poursuites devant la Cour pénale internationale ${ }^{54}$ ou devant un tribunal spécial, lorsqu'il est constitué, et donc de donner droit à des réparations s'il est prouvé qu'un État ayant reconnu la compétence de la Cour a échoué à traduire les criminels en justice, soit volontairement, soit par

$46 \quad$ Ibid, art 8.

47 Sorel, supra note 19 à la p 127.

48 Convention de Genève pour l'amélioration du sort des blessés et des malades dans les forces armées en campagne, 12 août 1949, 75 RTNU 31 (entrée en vigueur : 21 octobre 1950) [Convention de Genève I]; Convention de Genève pour l'amélioration du sort des blessés, des malades et des naufragés des forces armées sur mer, 12 août 1949, 75 RTNU 85 (entrée en vigueur : 21 octobre 1950) [Convention de Genève II]; Convention de Genève relative au traitement des prisonniers de guerre, 12 août 1949, 75 RTNU 135 (entrée en vigueur: 21 octobre 1950) [Convention de Genève III]; Convention de Genève relative à la protection des personnes civiles en temps de guerre, 12 août 1949, 75 RTNU 287 (entrée en vigueur : 21 octobre 1950) [Convention de Genève IV].

49 Nicolas Michel et Katherine Del Mar, «Opérations de maintien de la paix et droit des conflits armés », dans Vincent Chetail, dir, Permanence et mutations du droit des conflits armés, Bruxelles, Bruylant, 2013, 185 à la p 189; Nations Unies, Opérations de maintien de la paix des Nations Unies : principes et orientations, 2008 aux pp 14-15, en ligne : <www.un.org> [Doctrine Capstone].

50 Pour plus de précisions sur la qualification des conflits armés et la participation des opérations de maintien de la paix, voir Sylvain Vité, « Typologie des conflits armés en droit international humanitaire : concepts juridiques et réalités » (2009) 91:873 RICR 69 aux pp 88-89 [Vité].

51 Licéité de la menace ou de l'emploi d'armes nucléaires, Avis consultatif, [1996] CIJ rec 226 au para 79 [Licéité de la menace]; réitéré par la Cour dans Conséquences juridiques de l'édification d'un mur dans le territoire palestinien occupé, Avis consultatif, [2004] CIJ rec 136 au para 157.

52 Statut de Rome, supra note 7 art $7 \mathrm{~g}$ ); Convention de Genève IV, supra note 48.

53 Henckaerts et Doswald-Beck, supra note 8.

$54 \quad$ Statut de Rome, supra note 7 art 5. 
incapacité 55 . Mais si théoriquement la Cour pénale parait être une solution, ce n'est pas le cas en pratique. Outre la problématique de l'étendue des immunités dont bénéficient les Casques bleus et la complexe procédure de leur levée ${ }^{56}$, nous verrons que l'ONU, elle aussi, est protégée par des immunités. Il apparaît donc complexe, voire impossible, pour une cour de justice de condamner l'organisation à indemniser les victimes des agissements des Casques bleus. Il faut toutefois rappeler que les soldats des OMPs ne sont pas toujours appelés à prendre part aux combats même lorsqu'ils sont déployés pendant un conflit armé ${ }^{57}$. En effet, lorsqu'une OMP est créée par le Conseil de Sécurité sur une base autre que celle prévue au chapitre 7, qui prévoit l'emploi de la force armée ${ }^{58}$, celle-ci ne sera pas considérée comme étant une partie au conflit et ne sera donc pas soumise aux règles du DIH, même déployée dans le cadre d'un conflit armé59. Les violences sexuelles restent néanmoins une infraction en tout temps au Code de conduite des Casques bleus ${ }^{60}$. Ainsi, «les praticiens du maintien de la paix des Nations Unies doivent avoir une bonne connaissance des principes et des règles du droit international humanitaire et les observer dans les situations où ils s'appliquent $»^{61}$. À cela, la Doctrine Capstone - document interne aux Départements des opérations de maintien de la paix et de l'appui aux missions qui définit la nature, la portée, les fonctions essentielles des OMPs ainsi que les principes auxquelles elles doivent se soumettre - ajoute que :

[1]e personnel d'une opération de maintien de la paix des Nations Unies, qu'il soit militaire, policier ou civil, devrait se conduire en conformité avec le droit international en matière des droits de l'homme et comprendre le rapport entre les tâches qui lui sont confiées et les droits de l'homme. Il incombe au personnel d'une opération de maintien de la paix des Nations Unies de tout faire pour éviter les violations des droits de l'homme. Il doit être en mesure de reconnaitre les abus et les violations des droits de

55 Sarah Pellet, «Cour pénale internationale» dans Encyclopadia Universalis, en ligne : $<$ www.universalis-edu.com.proxy.bibliotheques.uqam.ca:2048/encyclopedie/cour-penaleinternationale $>$.

56 Pour plus de détails sur la levée des immunités, voir Modèle d'accord sur le statut des forces pour les opérations du maintien de la paix, Doc off AG NU, 1990, Doc NU A/45/594 6 au para 47 [Modèle d'accord]; Convention sur les privilèges, supra note 31 (la levée des immunités pose des problèmes au niveau politique car si l'ONU lève les immunités des Casques bleus, les États peuvent décider de ne plus fournir de contingents. Or, l'ONU n'ayant pas de force armée qui lui est propre, elle reste dépendante des États contributeurs de troupes. Se pose également le problème lié aux États dans lesquels les OMPs sont déployées. Ces derniers sont souvent des États qui ne respectent pas toujours les droits de l'homme. Ainsi, retirer les immunités du personnel onusien viendrait à l'exposer à des atteintes à ses droits, ce qui serait contraire à l'art 1(3) de la Charte. Ceci explique la procédure lente et complexe d'enquête avant de retirer une immunité). Voir Marco Odello et Róisín Burke, « Between Immunity And Impunity: Peacekeeping And Sexual Abuses And Violence » (2016) 20:6 Int'1 JHR 839 aux pp 845-849.

57 Vité, supra note 50 à la p 88; Doctrine Capstone, supra note 49 à la p 34.

58 ONU, Mandats et fondements juridiques des opérations, en ligne : <www.un.org/fr>.

59 Rapport Ban, supra note 22 aux paras 75-80.

60 United Nations, Conduct and discipline unit, Ten Rules: Code of Personal Conduct For Blue Helmets, art 4, en ligne : <www.un.org/en/peacekeeping/documents/ten_in.pdf>; Circulaire $d u$ Secrétaire général : Dispositions spéciales visant à prévenir l'exploitation et les abus sexuels, Doc off AG NU, 2005, Doc NU ST/SGB/2003/13.

61 Circulaire du Secrétaire général sur le respect du droit international humanitaire par les forces des Nations Unies, Doc off CES NU, Doc NU ST/SGB/1999/13 (1999) art 3. 
l'homme et se tenir prêt à intervenir de manière appropriée, tout en restant dans les limites de son mandat et de ses compétences. Le personnel d'une opération de maintien de la paix des Nations Unies doit respecter les droits de l'homme dans ses rapports avec ses collègues et la population locale ainsi que dans sa vie publique et privée. Ceux qui commettent des abus doivent être tenus pour responsables de leurs actes. ${ }^{62}$

Enfin, cette Doctrine Capstone fait mention des facteurs de réussite d'une OMP que sont la légitimité et la crédibilité de la mission, impliquant que «les missions doivent appliquer une politique de tolérance zéro envers l'exploitation et les abus sexuels, ainsi que toute autre forme grave de mauvaise conduite ${ }^{63}$, ces cas devant être traités avec « justesse et fermeté ${ }^{64}$.

Cependant, dans l'hypothèse où le DIH ne serait pas applicable, il reste les droits de l'homme qui, eux, s'appliquent en tout temps, qu'il y ait un conflit ou non. Comme l'a rappelé la Cour internationale de justice, l'application des droits de l'homme ne cesse pas en temps de guerre et la protection des civils doit rester primordiale $^{65}$. Ainsi, même si la protection des civils au travers du DIH est impossible, le personnel des OMPs reste soumis au respect des droits de l'homme, autant en tant qu'agent des États contributeurs qu'en tant que membre d'une OMP. Ce sont d'ailleurs des droits que l'ONU se targue de faire respecter ${ }^{66}$. Or, si les instruments relatifs aux droits de l'homme ne prévoient pas spécifiquement l'interdiction du viol et des violences sexuelles, ils le font implicitement au travers de la protection de l'intégrité physique ${ }^{67}$. Ainsi, la Déclaration universelle des droits de $l^{\prime}$ homme $^{68}$ prévoit à son article 5 que «[n]ul ne sera soumis à la torture, ni à des peines ou traitements cruels, inhumains ou dégradants $\gg{ }^{69}$. Cette interdiction de la torture a également été reconnue comme norme de jus cogens par le Tribunal pénal international pour l'ex-Yougoslavie en ce que cette interdiction constitue «l'une des normes les plus fondamentales de la communauté internationale $»^{70}$. Le droit à l'intégrité physique est également prévu à l'article $7 \mathrm{du}$ Pacte international relatif aux droits civils et politiques ${ }^{71}$, mais également par de nombreux traités régionaux comme

62 Doctrine Capstone, supra note 49 à la $\mathrm{p} 14$.

63 Ibid à la p 40.

64 Ibid.

65 Licéité de la menace, supra note 51 au para 25 et ss.

66 Charte des NU, supra note 14 arts 1(3), 55 al c- 56.

${ }^{67}$ La jurisprudence internationale a reconnu la valeur de droit coutumier de ce droit dans Jugement Furundzija, supra note 7 au para 170. Voir Comité pour l'élimination de la discrimination à l'égard des femmes, Constatations du Comité pour l'élimination de la discrimination à l'égard des femmes présentées en application du paragraphe 3 de l'article 7 du Protocole facultatif à la Convention sur l'élimination de la discrimination à l'égard des femmes, Doc off NU, 46 ${ }^{\mathrm{e}}$ sess, Doc NU CEDAW/C/46/D/18/2008 (2010) au para 8.7; Comité des droits de l'homme, Constatations du Comité des droits de l'homme au titre du paragraphe 4 de l'article 5 du Protocole facultatif se rapportant au Pacte international relatif aux droits civils et politiques, Doc off NU, $107^{\mathrm{e}}$ sess, Doc NU CCPR/C/107/D/1945/2010 (2013).

68 Déclaration universelle, supra note 11.

69 Ibid, art 5.

70 Jugement Furundzija, supra note 7 au para 154.

71 PIDCP, supra note 11 , art 7. 
la Convention américaine relative aux droits de l'homme ${ }^{72}$; la Convention de sauvegarde et de protection des droits de l'homme ${ }^{73}$ ou encore la Charte africaine des droits de l'homme et des peuples ${ }^{74}$.

\section{B. La mise en pratique par l'ONU de l'obligation de réparer}

Dans le cadre des OMPs, la diversité des acteurs d'un différend implique que le litige puisse être résolu sur le fondement de droits différents ${ }^{75}$. L'ONU applique donc son mode de règlement au cas par cas, c'est-à-dire que, pour chaque différend, elle cherche le droit applicable puis le mécanisme de règlement qui va s'appliquer ${ }^{76}$. En effet, il faut savoir qu'une OMP fait intervenir une grande diversité d'acteurs allant du personnel volontaire aux militaires en passant par les fonctionnaires et les policiers ${ }^{77}$. Tous ne sont pas sous le même régime juridique. De plus, les infractions à caractère sexuel ont des qualifications différentes en droit international ou en droit national. En droit pénal international et en DIH par exemple, le viol peut prendre plusieurs qualifications selon les faits réunis : il peut être considéré comme un crime de génocide $^{78}$, un crime contre l'humanité ${ }^{79}$, un crime de guerre ${ }^{80}$ ou encore une violation des lois et coutumes de la guerre ${ }^{81}$. Mais on l'a vu, ces branches du droit international ne sont pas toujours applicables aux membres des OMPs et leur application dépend de beaucoup de conditions comme la levée des immunités, la qualification du conflit armé, le rôle de cette OMP dans ce conflit, et surtout la compétence de la Cour pénale internationale. La Convention sur les privilèges et immunités vient donc pallier cette difficulté en précisant que «l'Organisation des Nations Unies devra prévoir des modes de règlements appropriés pour les différends en matière de contrats et autres différends de droit privé dans lesquels l'Organisation serait partie $\|^{82}$, afin qu'un accès direct à la justice, dans son sens le plus large, soit offert aux personnes privées. À ce titre, l'ONU a donc souvent recours à la pratique de l'arbitrage et souscrit à des clauses compromissoires ${ }^{83}$ lorsqu'elle conclut des

72 Convention américaine relative aux droits de l'homme, 22 novembre 1969, 1144 RTNU 182 art 5 (entrée en vigueur : 18 juillet 1978).

73 Convention européenne des droits de l'homme, supra note 11, art 3.

74 Conférence des chefs d'États et du gouvernement, Charte africaine des droits de l'homme et des peuples, 27 juin 1981, 1520 RTNU 217 arts 4-5 (entrée en vigueur : 21 octobre 1986).

75 Mathias Forteau, «Régime général de responsabilité ou lex specialis? » (2013) 46:1 Rev b dr Intern 147 à la $\mathrm{p} 153$.

76 Ascensio, supra note 36 à la p 1124 .

77 ONU, Opérations de maintien de la paix, « Les questions soulevées par les opérations de maintien de la paix », en ligne : <www.un.org/fr/peacekeeping/issues/index.shtml>; Odello, supra note 30 à la p 366.

78 Jugement Akayesu, supra note 7 aux paras 731-32. Confirmé par le Jugement Musema, supra note 7 au para 933.

79 Statut de Rome, supra note 7.

80 Jugement Furundzija, supra note 7 au para 172.

81 Ibid.

82 Convention sur les privilèges, supra note 31 , art 8.

83 Jacqueline Barbin, "Compromis et clause compromissoire» dans Encyclopaedia Universalis, en ligne : <www.universalis.fr/encyclopedie/compromis-et-clause-compromissoire/>. 
contrats avec des personnes privées ${ }^{84}$, ce qui supprime le problème de l'immunité de juridiction $^{85}$. Néanmoins, cette pratique reste presque exclusivement réservée aux différends contractuels de l'organisation.

Pour le cas particulier des OMPs, l'ONU prévoit des commissions de réclamations dans l'accord qu'elle conclut avec l'État hôte de l'opération ${ }^{86}$. Le Modèle d'accord sur le statut des forces ${ }^{87}$ prévoit que cette commission doit comprendre trois entités, soit un membre désigné par l'ONU, un membre désigné par le pays hôte et un troisième désigné d'un commun accord entre l'ONU et le pays hôte de $1^{\prime} \mathrm{OMP}^{88}$ pour assurer la plus grande neutralité possible. Cette commission choisit sa propre procédure et rend des décisions obligatoires et définitives à moins qu'une interjection en appel ne soit autorisée par le Secrétaire général et le gouvernement concerné au moment de l'élaboration du statut. En cas d'appel, l'instance est un tribunal arbitral ${ }^{89}$.

Selon Jean-Marc Sorel ${ }^{90}$, c'est un système proche de l'arbitrage que l'on peut retrouver dans plusieurs opérations comme l'Opération des Nations Unies au Congo ou la Mission des Nations Unies pour l'assistance au Rwanda (MINUAR). Néanmoins, ces commissions ont peu fonctionné en pratique en raison d'un manque d'efficacité ${ }^{91}$. Sorel explique qu' « une telle commission était suggérée par l'article 50 de l'Accord entre l'ONU et le Rwanda du 5 novembre 1993 pour la MINUAR, mais l'ONU a refusé sa création préférant passer par le traitement du Comité consultatif $[\ldots] »^{92}$. L'inconvénient est que l'on passe alors d' « un système quasi-juridictionnel à un système unilatéral qui nie le principe du contradictoire $»^{93}$. En effet, au détriment des commissions de réclamations, la pratique préfère s'orienter vers un mécanisme non contentieux, purement interne à l'ONU, qui consiste à faire examiner par un comité local les demandes d'indemnisation présentées par des tiers pour préjudice corporel, décès, perte de biens ou dommages matériels, imputables à des actes commis par des éléments civils ou militaires de la mission dans l'exercice de leurs fonctions officielles, et à les faire approuver ou formuler des propositions de règlements ${ }^{94}$.

84 Christian Dominicé, «Observations sur le contentieux des Organisations internationales avec des personnes privées » (1999) 45:1 AFDI 623 à la p 642 [Dominicié].

85 Ibid à la p 643.

$86 \quad$ Ibid.à la p 645; Modèle d'accord, supra note 56 au para 51.

87 Ibid.

88 Ibid.

89 Modèle d'accord, supra note 56 au para 51 ; Sorel, supra note 19 à la p 131.

90 Sorel, supra note 19 à la $\mathrm{p} 134$.

91 Financement de la force de protection des Nations Unies, de l'opération des Nations Unies pour le rétablissement de la confiance en Croatie, de la force de déploiement préventif des Nations Unies et du quartier général des forces de paix des Nations Unies, Doc off AG NU, 1996, Doc NU A/51/491 (1996) au para 9; Zwanenburg, supra note 16 à la p 28.

92 Sorel, supra note 19 à la p 134.

93 Ibid.

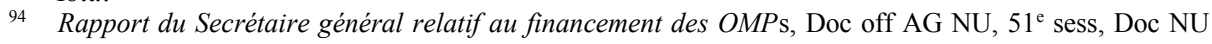
A/51/389 (1996) aux para 22-23 [Rapport OMPs]. 
Néanmoins, ces comités locaux constituent des organes de l'ONU et grand est le risque de contrevenir au principe selon lequel «nul ne peut être juge et partie $»^{95}$. Partant de là, ils ne contribuent pas à ce que les victimes perçoivent la justice comme rendue à leur égard. C'est pourquoi il est malheureux que les commissions de réclamations ne fonctionnent plus. Avec un système tripartite qui comprend une personne nommée communément par l'ONU et l'État hôte, le système pourrait être plus objectif.

Le problème majeur dans ce domaine reste que même si l'ONU ne recule pas devant sa responsabilité pour certains actes commis au cours des OMPs, la méthode de règlement des conflits est encore empreinte de " confidentialité et d'ambiguïté ${ }^{96}$, mais aussi d'un pouvoir discrétionnaire. En effet, au lieu de mettre en place des mécanismes de droit commun, l'ONU préfère passer par le règlement à l'amiable qui n'est pas encadré par des textes. Cela implique que les négociations ne sont pas publiées et que l'on ne peut connaître le détail de ces règlements. Ainsi, cela place l'ONU dans une position de force face aux victimes. Lorsqu'un arrangement n'est pas conclu, la victime se heurte à l'immunité de l'ONU devant les juridictions nationales et n'a donc pas beaucoup de choix : accepter l'indemnisation de l'organisation, quand bien même elle serait insuffisante compte tenu du préjudice subi, ou ne rien obtenir du tout. Ce mécanisme place les victimes au deuxième plan dans le processus de plainte et d'indemnisation. Force est de constater que ce système vise surtout à prouver que 1'ONU a mis des mécanismes en place ${ }^{97}$ sans vraiment s'interroger sur leur efficacité ou leur accessibilité ${ }^{8}$. En effet, un rapport du Secrétaire général en date de 2009 fait état de difficultés dans l'implantation des mécanismes sur le terrain, notamment en raison d'un manque d'uniformité des procédures, mais également d'un manque d'adaptation aux cultures locales pour lesquelles, bien souvent, les agressions sexuelles sont perçues comme des événements honteux jetant l'opprobre sur les victimes ${ }^{99}$. Ainsi, si l'ONU a bel et bien mis en place des mécanismes pour recenser les plaintes des victimes présumées d'agressions sexuelles par des membres des OMPs, le fonctionnement de ces derniers nécessite de l'engagement, beaucoup de temps et d'importantes ressources financières ${ }^{100}$. C'est pour cette raison que l'ONU n'a pas encore pris la peine de les améliorer et les a délaissés au profit de mécanismes officieux, lui permettant notamment de ne pas se départir de son immunité.

95 Convention européenne des droits de l'homme, supra note 11 art 6; Déclaration universelle, supra note 11, art 10; PIDCP, supra note 11 art 14(1); Zwanenburg, supra note 16 à la p 28; Bode, supra note 33 aux pp 771-772.

96 Sorel, supra note 19 à la p 128; Ascensio, supra note 36 à la p 1143.

97 Stratégie globale d'aide et de soutien aux victimes d'actes d'exploitation ou d'agression sexuelles commis par des membres du personnel des Nations Unies ou du personnel apparenté, Doc off AG NU, $62^{\mathrm{e}}$ sess, Doc NU A/RES/62/214 (2008) à la p 2. À ce sujet, voir ONU, « Conduct in UN field missions », en ligne : <cdu.unlb.org/UNStrategy/RemedialAction.aspx $>$.

98 Implementation of the United Nations Comprehensive Strategy on Assistance and Support to Victims of Sexual Exploitation and Abuse by United Nations Staff and Related Personnel, Doc off AG NU, $64^{\mathrm{e}}$ sess, Doc NU A/64/176 (2009) aux para 55-65.

$99 \quad$ Ibid au para 60.

100 Ibid au para 72. 


\section{Les failles des mécanismes onusiens : entre immunités et manque de transparence}

Si l'ONU dispose bel et bien de mécanismes de réparation, il apparaît que ce système repose entièrement sur le bon vouloir de l'ONU à reconnaître sa responsabilité ou non (A) et que, par ailleurs, les victimes des actes commis par les Casques bleus n'ont souvent qu'un rôle secondaire dans le processus de règlement (B).

\section{A. Les difficultés liées aux immunités de l'Organisation des Nations Unies $^{101}$}

L'ONU peut être tenue responsable au regard du droit international ${ }^{102}$. Cette possibilité a été évoquée dans un avis consultatif de la Cour internationale de justice affirmant que l'organisation était responsable des actes commis par ses agents dans le cadre de leurs fonctions ${ }^{103}$. Il faut alors distinguer les différends de nature contractuelle et ceux de nature extracontractuelle. Pour ce qui est des contrats, l'ONU peut renoncer unilatéralement à se prévaloir de son immunité. C'est notamment le cas pour ses polices d'assurance. Lorsque l'ONU se défait de son immunité, le tiers lésé a la possibilité d'agir devant les juridictions étatiques et c'est l'assureur de l'organisation qui agira en tant que défendeur ${ }^{104}$. La pratique veut que l'ONU utilise souvent cette technique lorsqu'elle se trouve dans une situation bilatérale. Or, traditionnellement, pour qu'un contrat soit signé, les deux parties doivent avoir trouvé un terrain d'entente ${ }^{105}$. Ainsi, l'ONU trouve un certain intérêt à renoncer à son immunité dans cette situation.

En revanche, pour les différends qui l'opposent à une personne privée (différends extracontractuels), les tribunaux n'ont pas compétence pour juger de l'affaire ${ }^{106}$. Qui plus est, puisqu'ils sont incompétents, ils ne peuvent ordonner à l'ONU de renoncer à ses immunités ${ }^{107}$, d'autant plus que, comme il a été mentionné plus haut, l'organisation est beaucoup plus encline à utiliser le règlement à l'amiable pour ce genre de différends, évitant ainsi la surmédiatisation d'un sujet déjà sensible et tabou. Dès lors que l'arrangement à l'amiable n'est pas satisfaisant pour la personne privée, celle-ci devrait pouvoir se tourner vers un juge. Toutefois, les immunités créent un vide juridique et une violation du droit d'accès à un juge ${ }^{108}$, un

\footnotetext{
101 Charte des NU, supra note 14, art 105(1).

102 Réparation des dommages subis au service des Nations Unies, Avis consultatif, [1949] CIJ rec 174; Zwanenburg, supra note 16 à la p 25.

103 Différend relatif à l'immunité de juridiction d'un rapporteur spécial de la Commission des droits de l'homme, Avis consultatif, [1999] CIJ rec 62 au para 66 [Différend relatif à l'immunité].

104 Ascensio, supra note 36 à la p 1128.

105 Ibid.

106 Différend relatif à l'immunité, supra note 103.

107 Stitching Mothers of Srebrenica et autres c Pays-Bas, n ${ }^{\circ}$ 65542/12, [2013] CEDH 1, 57 EHRR SE10 aux paras 163-64 [Mothers of Srebrenica].

108 Convention européenne des droits de l'homme, supra note 11, art 6(1), 13.
} 
principe de droit coutumier ${ }^{109}$, ce qui entraîne une violation aux droits de l'homme et notamment à l'ensemble des règles liées au droit au procès équitable ${ }^{110}$. Or, en 2005 , l'Assemblée générale de l'ONU a rappelé l'importance du droit à la réparation et donc à la possibilité pour les victimes d'avoir un recours ${ }^{111}$.

L'idée ici n'est pas de rejeter les immunités dont bénéficie l'ONU. Leur utilité est indéniable pour assurer l'indépendance de l'organisation et la mise en œuvre des différentes missions qu'elle doit exercer, comme c'est notamment le cas pour les $\mathrm{OMPs}^{112}$. Le problème qui se pose réside dans l'étendue de ces immunités qui sont assez larges pour couvrir la diversité des activités mises en œuvre au cours d'une OMP ${ }^{113}$. L'ONU ne doit néanmoins pas confondre immunité et impunité et pour cela, il conviendrait donc plutôt de limiter ces immunités au cadre strict des actes nécessaires à la réalisation de sa mission, comme c'est le cas par exemple des chefs d'États ${ }^{144}$ ou pour les membres des OMPs ${ }^{115}$.

Qui plus est, ce droit d'accès au juge est sujet à des inégalités selon que la personne lésée est liée à l'ONU par un contrat ou non. Dans le premier cas, lorsqu'en contradiction avec les dispositions prises par le contrat, l'ONU fait valoir son immunité et qu'il n'y a pas de possibilité de recourir à des modes alternatifs de règlement des conflits, la personne liée par un contrat peut saisir le juge national ${ }^{116}$. Par contre, s'agissant de la responsabilité extracontractuelle de l'ONU, la jurisprudence est beaucoup plus ferme : il n'appartient pas aux juges nationaux de traiter des différends entre une personne privée et une organisation internationale ${ }^{117}$. Également, la Cour européenne des droits de l'homme a, dans la même lignée, affirmé que le refus d'un État de lever l'immunité de juridiction de l'ONU pour connaître des demandes en réparation n'est pas une violation des articles 6 et 13 de la Convention européenne des droits de l'homme concernant le procès équitable et le droit à un recours effectif ${ }^{118}$, notamment en raison du fait qu'il existerait des « limitations implicitement admises » à ce droit d'accès au juge, appelant, « de par sa

109 Ascensio, supra note 36 à la p 1129.

110 Voir TAOIT, Klausecker c. OEB, n² 2657, 11 juillet 2007, au para 6; Golder c Royaume-Uni (1975), 18 CEDH Sér A [Golder].

111 Principes fondamentaux et directives, supra note 40.

112 Dominicié, supra note 84 à la p 639; Beer et Regan c Allemagne, no 28934/95, [1999] CEDH 1.

113 Laure Milano, « Les immunités issues du droit international dans la jurisprudence européenne » (2008) 76 Revue trimestrielle des droits de l'Homme 1059 à la p 1065 [Milano].

114 Affaire relative au mandat d'arrêt du 11 avril 2000 (République démocratique du Congo c Belgique), [2002] CIJ rec 3 au para 61.

115 Aspects administratifs et budgétaires du financement des opérations de maintien de la paix des Nations Unies : financement des opérations de maintien de la paix des Nations Unies, Doc off AG NU, 1997, Doc NU A/51/967 [Aspects administratifs et budgétaires]; Mémorandum d'accord type relatif aux contributions conclu entre l'Organisation des Nations Unies et l'État participant fournissant des ressources à l'opération de maintien de la paix des Nations Unies, Doc off AG NU, $51^{\mathrm{e}}$ sess, Doc NU A/51/967 (1997) art 9.

116 Justin Vanderschuren, «De quelques considérations sur les immunités octroyées aux organisations internationales » (2014) 9:6553 Journal des Tribunaux 145.

117 Différend relatif à l'immunité, supra note 103 au para 67 ; Bode, supra note 33 à la p 770.

118 Mothers of Srebrenica, supra note 107. 
nature même, une règlementation de l'État ${ }^{119}$. La principale raison du refus des cours internationales de reconnaître tant leur propre compétence qu'une violation du droit d'accès au juge dans le traitement des relations entre les organisations internationales et les personnes privées est qu'elles prendraient le risque de se voir submergées de requêtes, ce qui allongerait la durée des affaires ${ }^{120}$, qui est déjà très longue ${ }^{121}$.

Pourtant, selon Laure Milano ${ }^{122}$, la Cour européenne des droits de 1'homme avait établi trois critères pour délimiter l'application des immunités internationales : la spécificité de la fonction, la spécificité de l'emploi couvert par l'immunité et l'existence d'une compensation à l'exonération de responsabilité ${ }^{123}$. L'objectif était de trouver un équilibre entre les intérêts des organisations à conserver leur immunité et la satisfaction du droit d'accès au juge pour les individus. Ces critères n'ont malheureusement pas été mis en pratique ${ }^{124}$.

On peut donc constater que les personnes privées lésées par un acte commis par l'ONU dépendent du bon vouloir de cette dernière à utiliser les modes alternatifs de règlement des conflits et à proposer une solution acceptable. Toutefois, celles qui souhaitent plutôt utiliser la voie judiciaire sont tributaires de la bonne volonté de l'ONU à se départir de son immunité ou non. Nous en avons une preuve avec la situation en Haïti où l'ONU refuse, pour le moment, de se départir de son immunité pour reconnaître sa part de responsabilité dans l'épidémie de choléra qui a infecté le pays après l'arrivée des Casques bleus népalais ${ }^{125}$.

\section{B. La place accordée à la victime dans les mécanismes d'indemnisation}

Comment les nationaux peuvent-ils poursuivre l'ONU, sachant que personne n'a, a priori, compétence pour régler les différends entre l'ONU et les personnes privées? Le processus dépend entièrement de la bonne volonté de l'organisation qui détient un pouvoir discrétionnaire dans la création des mécanismes. $\mathrm{Si}$, comme il a été vu précédemment, des moyens d'indemnisation sont bel et bien offerts par l'ONU, peu de démarches sont faites en revanche dans l'intention d'inclure la victime dans le processus. En effet, la procédure observée par les comités locaux reste assez complexe ${ }^{126}$.

119 Golder, supra note 110 aux para 36-38.

120 Milano, supra note 113 à la $\mathrm{p} 1068$.

121 S'agissant des cours de justice des États hôtes des opérations de maintien de la paix, il convient également de souligner que bien souvent, l'état du système judiciaire ne permet pas d'offrir la possibilité d'un recours aux victimes. Voir Zwanenburg, supra note 16 à la p 39.

122 Milano, supra note 113 à la p 1085.

123 Dyer c Royaume-Uni (1984), CEDH (Sér A) 39 à la p 246.

124 Ibid.

125 Pour plus de précisions sur cette affaire, voir Gustavo Gallón, Rapport de l'Expert indépendant sur la situation des droits de l'homme en Haïti, Doc off AG NU, 25 $5^{\mathrm{e}}$ sess, Doc NU A/HRC/25/71 (2014) au para 76; Bode, supra note 33 aux pp 778 et ss.

126 Si l'indemnité est approuvée par le comité d'examen des demandes d'indemnisation, le groupe pour les demandes d'indemnisation offre l'indemnité déterminée à la personne lésée. Une décharge est alors 
En outre, s'agissant de l'indemnisation des dommages causés aux tiers par les membres du personnel de maintien de la paix dans l'exercice de leurs fonctions, la résolution de l'Assemblée générale de $1^{\prime} \mathrm{ONU}^{127}$ prévoit de restreindre la prescription à six mois pour le demandeur ${ }^{128}$; elle prévoit également que «[1]e montant de l'indemnité due en cas de préjudices corporels subis par un individu, ou de maladie ou de décès de l'intéressé, [...] ne pourra dépasser 50000 dollars des ÉtatsUnis $[\ldots] \gg{ }^{129}$. Comble de toutes ces restrictions, la résolution prévoit que l'ONU n'indemnise pas le préjudice moral, mais uniquement le préjudice matériel ${ }^{130}$ causé par un acte nécessaire à l'exécution de la mission de l'OMP, ou par le personnel de l'opération dans le cadre de ses fonctions ${ }^{131}$.

Par ailleurs, le problème qui se pose est également politique, car une formalisation des procédures d'indemnisation « risque de mettre en évidence de façon embarrassante la responsabilité des États membres autant que celle de l'ONU $\gg^{132}$. Dans un souci de restaurer son image et sa légitimité, l'ONU n'accorde à la victime qu'un rôle secondaire dans le processus, ce qui ne favorise pas la réconciliation que l'organisation prétend vouloir apporter grâce à ses opérations de maintien de la paix $^{133}$. Certes, l'ONU a marqué sa volonté d'accepter sa responsabilité en la matière. La Résolution 52/247 134 en est la preuve. Elle a montré « l'intention claire d'élaborer un régime spécifique aux Nations Unies pour l'indemnisation des dommages et préjudices subis du fait des activités des OMP $\gg{ }^{135}$. Le paradoxe de cette résolution est qu'en même temps qu'elle reconnaît sa responsabilité pour les dommages causés par ses membres, l'ONU restreint sa propre responsabilité ${ }^{136}$.

signée par cette dernière, dégageant la responsabilité de l'ONU. Jusque-là tout paraît simple, mais la procédure se complexifie beaucoup plus lorsque l'indemnité qui est proposée dépasse les limites financières prévues. La demande est alors renvoyée au siège de l'ONU. Les recommandations d'indemnisation sont ensuite présentées à la «Division de l'administration et de la logistique des missions du Département des opérations de maintien de la paix qui les transmet à son tour à la Division du financement des opérations de maintien de la paix », soit le Bureau de la planification des programmes, du budget et des finances. Voir Rapport OMPs, supra note 94.

127 Demandes d'indemnisation, supra note 17.

128 Ibid au para 8.

129 Ibid au para $9(\mathrm{~d})$.

130 Ibid au para $9(\mathrm{~b})$.

131 «Liability of the United Nations for claims involving off-duty acts of members of peace-keeping forces - Determination of "off-duty" versus "on-duty" status » (1986) 24 United Nations Juridical Yearbook 300 à la p 300.

132 Ascensio, supra note 36 à la p 1140 .

133 Le manque d'implication de l'ONU dans la réparation des dommages subis du fait d'exactions sexuelles commises par les membres des opérations de maintien de la paix provoque à terme un réel problème de confiance et de coopération avec les populations locales. Qui plus est, le flagrant manque de respect des droits de l'homme, que l'ONU prêche pourtant de manière véhémente, soulève à nouveau la question de leur utilisation pour légitimer les actions des organisations internationales et des États plutôt que par réelle préoccupation des civils. À ce sujet, voir Rémi Bachand, « Le droit international et l'idéologie "droit de-l'hommiste" au fondement de l'hégémonie occidentale » (2014) HS RQDI 69 [Bachand].

134 Demandes d'indemnisation, supra note 17.

135 Pierre Bodeau-Livinec, «Les faux-semblants de la lex specialis : l'exemple de la résolution 52/247 de l'assemblée générale des Nations Unies sur les limitations temporelles et financières de la responsabilité de l'ONU » (2013) 46 Rev BDI 117 à la p 120.

136 Ibid à la p 126. 
Il faut savoir également que dans le cadre de cette résolution, l'ONU n'est responsable que des faits commis dans l'exercice de l'OMP et pas pour les actes privés des membres de l'opération ou ceux relevant d'une faute de leur part ${ }^{137}$ qu'ils auraient pu commettre hors service, de manière indépendante et à titre individuel ${ }^{138}$. Force est de constater qu'avec cette résolution, on peut effectivement dire qu'il y a une volonté de la part de l'ONU d'accepter sa responsabilité pour les dommages causés par les membres des OMPs. Néanmoins, on s'aperçoit que la Résolution 52/247 ne s'applique pas à l'indemnisation des victimes d'exactions sexuelles. L'ONU pourrait néanmoins indemniser ce genre de dommage ${ }^{139}$, mais n'étant pas établi textuellement dans la résolution, cela reste soumis à sa bonne volonté. Or, comme l'a dit l'assistant exécutif du Général Roméo Dallaire, le Major Brent Beardsley: "le massacre ça tue le corps, mais le viol ça tue l'âme ${ }^{140}$. Comment les victimes de ces exactions peuvent-elles alors tenter de reconstruire une vie si, en plus de faire face au rejet de leurs familles, à des blessures physiques et pour certaines à la contamination au VIH, l'ONU ne prend pas en compte les douleurs, tant physiques que morales, qui leur ont été infligées?

Pourtant, si nous reprenons notre analyse, nous savons que l'ONU affirme exercer ses missions dans le respect des droits de l'homme ${ }^{141}$. Elle affirme non seulement que toute personne lésée a le droit d'obtenir réparation pour le préjudice subi $^{142}$, mais aussi que

résoudre le problème de l'exploitation et des abus sexuels imputables à des personnels de maintien de la paix des Nations Unies est la responsabilité commune de l'Organisation et des États Membres, et seule une action résolue de la part à la fois du Secrétariat et des États Membres permettra d'y arriver. ${ }^{143}$

Certains auteurs, comme Bodeau-Livinec ${ }^{144}$, semblent penser que l'ONU ne pratique pas ce qu'elle prêche puisqu'elle ne propose pas vraiment de mécanismes de réparations adéquats pour les victimes d'exactions sexuelles commises par les membres du personnel de maintien de la paix. Partant de là, si effectivement on ne peut nier l'absence de sa responsabilité pénale directe dans ces affaires, elle est en revanche responsable d'avoir failli à son obligation de respecter les droits de l'homme. Or:

[i]1 y a fait internationalement illicite d'une organisation internationale lorsqu'un comportement consistant en une action ou une omission :

137 Aspects administratifs et budgétaires, supra note 115 art 9.

138 Différend relatif à l'immunité, supra note 103 au para 66.

139 Ibid.

140 Le Procureur c Theoneste Ilagosora, ICTR-98-41-A, Jugement (14 décembre 2011) (Tribunal pénal international pour le Rwanda, Chambre d'appel).

141 Charte des NU, supra note 14, art 1(3).

142 Principes fondamentaux et directives, supra note 40.

143 Stratégie globale, supra note 42 à la p 2.

144 Bodeau-Livinec, supra note 41; Voir Zwanenburg, supra note 16; Bachand, supra note 133; Jean-Marc Coicaud, «Réflexions sur les organisations internationales et la légitimité internationale : contraintes, pathologies et perspectives » (2001) 170:4 R Intl Sciences Sociales 573. 
a) Est attribuable à cette organisation en vertu du droit international ; et

b) Constitue une violation d'une obligation internationale de cette organisation $^{145}$.

Cela implique que l'ONU, c'est-à-dire ses agents et ses organes, est « lié[e] par toutes les obligations que lui imposent les règles générales du droit international, son acte constitutif ou les accords internationaux auxquels [elle] est partie ${ }^{146}$. Dès lors que les victimes de viols et agressions sexuelles commis par les Casques bleus ne peuvent se voir proposer aucun mécanisme de réparation de la part de l'ONU, il apparaîtrait logique que l'organisation ait violé ses obligations. Par ailleurs, détenant un devoir de formation et de prévention sur les contingents envoyés dans les OMPs ${ }^{147}$, on peut déduire qu'elle a failli à ce devoir si des infractions sexuelles sont commises $^{148}$. Elle serait donc responsable d'avoir violé son obligation de protéger les populations, essence même de la mission de rétablissement, du maintien ou de l'imposition de la paix, en ne formant pas de façon optimale les contingents. C'est donc sur cette omission qu'il serait possible pour les victimes d'obtenir réparation du préjudice qu'elles ont subi.

Cette analyse permet de mettre en exergue la rareté des mécanismes en question et leur manque d'efficacité et d'effectivité quant à l'indemnisation des victimes d'exactions sexuelles commises par les membres des opérations de maintien de la paix. Le Rapport Zeid ${ }^{149}$ a donc suggéré des solutions pour pallier ce manque d'implication dans la résolution de ce type d'affaires comme nous le verrons rapidement en conclusion.

\section{$* * *$}

On peut donc malheureusement valider l'hypothèse selon laquelle en dépit du fait qu'il existe des mécanismes par le biais desquels une personne privée peut demander réparation pour un dommage subi par des membres des OMPs, leur efficacité et leur effectivité sont rendues nulles par le non-respect des droits de l'homme qui prévoient un droit d'accès au juge, par les immunités de l'ONU devant les juridictions nationales et par la non-utilisation pure et simple des mécanismes mis en place par l'organisation elle-même. Il faut également remarquer qu'aucune évolution n'a été constatée dans ce domaine, que ce soit du côté de l'organisation, dont les mécanismes n'ont pas évolué depuis le Modèle d'accord sur le statut des

145 Projet d'articles, supra note 16 art 4.

146 Ibid.

147 Stratégie globale, supra note 42 au para 38.

148 Le Secrétaire général lui-même reconnaît que certains facteurs propres à l'Organisation contribuent à la persistance des cas d'exploitation et d'atteintes sexuelles comme l'absence d'une formation harmonisée et systématique pour toutes les catégories de personnel, le manque de fermeté de la part des responsables du personnel civil et en tenue ou encore le manque d'attention de la part des hauts responsables de l'ONU et des États membres. Voir Dispositions spéciales, supra note 2 au para 12.

149 Stratégie globale, supra note 42. 
forces de $1990^{150}$, ou du côté de la jurisprudence qui reste inchangée depuis les premiers arrêts sur la question des immunités de l'ONU devant les juges nationaux rendus par les juridictions belges en 1966 et $1969^{151}$.

S'il faut bien reconnaître que l'ONU a fait preuve de bonne foi afin d'améliorer les mécanismes d'indemnisation offerts aux personnes privées lésées par une action commise dans le cadre d'une $\mathrm{OMP}^{152}$, les actions commises par les membres des opérations en dehors de l'exercice de leur mandat restent encore un problème. Deux choses sont à noter ici; premièrement, l'ONU détient une responsabilité vis-à-vis du personnel de maintien de la paix puisqu'elle doit le former et préparer le personnel mandaté sur place ${ }^{153}$. Elle doit également s'assurer qu'un cadre de vie adéquat et supportable est offert aux membres des OMPs, car il s'avère que plus le personnel s'ennuie et plus il vit dans des conditions difficiles, plus il sera enclin aux débordements ${ }^{154}$. Évidemment, il n'est pas question de trouver des excuses à ce genre d'actes, mais bien de s'assurer que le personnel envoyé l'est en toute connaissance du milieu difficile qui l'attend, étant donné que les OMPs sont souvent déployées dans des contextes conflictuels ${ }^{155}$. Bien que l'ONU n'ait pas elle-même commis ces actes abominables, elle reste responsable des formations en matière de droit de l'homme et de conduite qu'elle a dispensées au personnel mandaté ${ }^{156}$. Deuxièmement, les OMPs sont souvent déployées dans des États où la religion et les traditions ancestrales sont fortement présentes. À ce titre, il faut savoir que les victimes d'exactions sexuelles sont souvent rejetées par leur famille et leur mari ${ }^{157}$. En effet, dénoncer un viol est la preuve d'un courage immense de la part des victimes, même si la plupart préfèrent le cacher afin de continuer à vivre leur vie ${ }^{158}$. Aux vues des conséquences qui découlent d'un viol, outre les dommages psychologiques et physiques d'avoir été violées, la responsabilité de l'ONU prévue par la Résolution 52/247 apparait malheureusement bien faible du fait des limites qu'elle contient.

Enfin, le problème de la diversité des acteurs sur place pose également celui

150 Modèle d'accord, supra note 56.

151 Cour de première instance, Bruxelles, mai 1966, Mandelier c Nations Unies et Belgique (1966), Journal des Tribunaux n 4553 (Belgique); Cour d'appel de Bruxelles, Bruxelles, 15 septembre 1969, Mandelier c Nations Unies et Belgique (1969), ILR (Belgique).

152 Demandes d'indemnisation, supra note 17.

153 Zwanenburg, supra note 16 aux pp 30-31; Doctrine Capstone, supra note 49 aux pp 34-44; ONU, «Uniting our strengths for peace : politics, partnership and people », 16 juin 2015 à la p 60, en ligne : <peaceoperationsreview.org/wp-content/uploads/2015/08/HIPPO_Report_1_June_2015.pdf>.

154 Stratégie globale, supra note 42 au para 50.

155 À titre d'exemple, on peut entre autres citer la Mission de l'Organisation des Nations Unies en République Démocratique du Congo. Voir Stratégie globale, supra note 42 au para 2.

156 Ibid au para 38.

157 Si l'on a souvent tendance à parler de victimes féminines, il ne faut néanmoins pas oublier que de jeunes garçons peuvent également faire l'objet de ces exactions sexuelles. Voir à ce sujet Marie Deschamps, Lutter contre l'exploitation et les atteintes sexuelles commises par les soldats de la paix, 17 décembre 2017, en ligne : <www.un.org/News/dh/infocus/centafricrepub/Independent-ReviewReport-Fr.pdf>.

158 Pour plus d'informations à ce sujet, voir Human Rights Watch, La République Démocratique du Congo, la guerre dans la guerre. Violence sexuelle contre les femmes et les filles dans l'est du Congo, 20 juin 2002, en ligne: <www.hrw.org/fr/report/2002/06/20/la-guerre-dans-la-guerre/violencesexuelle-contre-les-femmes-et-les-filles-dans> à la p 14. 
de la diversité des régimes juridiques applicables et donc celui des enquêtes comme le soulève le rapport Zeid ${ }^{159}$. À ce titre, il convient de noter les efforts des États, pris individuellement, pour punir les actes scandaleux commis par leurs soldats, comme le Canada ${ }^{160}$, les États-Unis ${ }^{161}$ ou la France ${ }^{162}$. La justice française mène d'ailleurs toujours des enquêtes concernant l'implication des soldats de l'opération Sangaris dans le viol d'enfants mineurs à l'aéroport de Bangui ${ }^{163}$. Néanmoins, cela passe par des cours martiales nationales qui ne permettent pas aux victimes d'être parties à ces instances et dont les jugements sont confidentiels. Le sentiment de justice des victimes n'est donc pas satisfait.

Il faut tout de même mentionner les récentes avancées soutenues par le nouveau Secrétaire général, António Guterres, qui a publié un rapport très prometteur sur les nouvelles stratégies à adopter pour prévenir l'exploitation et les atteintes sexuelles. Il propose une stratégie qui replace les victimes au centre du processus de répression et de réparation, notamment en confiant à un «fonctionnaire [...] les fonctions de défense des droits des victimes sur le terrain $»^{164}$. Ensuite, il réaffirme vouloir promouvoir la transparence des procédures judiciaires et administratives, instaurer un « réseau multipartite » devant prendre en compte les dirigeants locaux et enfin refonder les mécanismes de prévention et la politique de sensibilisation et de déstigmatisation des victimes d'exploitation et d'atteintes sexuelles ${ }^{165}$. Ce rapport soutient également la participation accrue des femmes aux opérations de maintien de la paix ${ }^{166}$, ce qui permet souvent de réduire les risques d'exploitation et d'atteintes sexuelles ${ }^{167}$.

L'Assemblée générale soutient également la mise en place d'un «fonds d'affectation spéciale pour financer les services spécialisés dont ont besoin les victimes d'exploitation sexuelle ou de violences sexuelles $»^{168}$ alimenté par les contributions volontaires. Il ne faut pas nier l'avancée de cette nouvelle idée, néanmoins elle place une nouvelle fois l'État en premier responsable des violations commises et non l'ONU qui, même si elle montre son implication, n'affirme pas sa responsabilité. À nouveau, nous tombons dans cette culture de l'impunité, ce qui relègue une fois de plus le respect des droits de l'homme et la transparence au bas de la liste des principes de l'ONU.

159 Stratégie globale, supra note 42 aux para 28-34.

160 Loi sur la défense nationale, LRC 1985, c N-5, art 130; R c Boland (1995) CMAC 374; Matchee c Canada (Procureur Général) 1999 CanLII 7478 (CF).

161 United States v Ronghi, 27 mai 2003, No. ARMY 20000635 (A. Ct. Crim. App.), et United States v Ronghi, 30 juin 2004, No. 03-0520, 60 MJ 83.

162 Cécile Bouanchaud, "Soupçons de viols en Centrafrique : que dit l'enquête ? », Europe 1 (30 avril 2015), en ligne : <www.europe1.fr/faits-divers/centrafrique-que-sait-on-des-soupcons-d-abus-sexuelsdans-1-armee-2441541>.

163 Nathalie Guibert, " Centrafrique : la justice écarte des accusations contre les soldats de "Sangaris" », Le Monde (5 janvier 2017), en ligne : <www.lemonde.fr/international/article/2017/01/05/centrafriquela-justice-ecarte-des-accusations-contre-les-soldats-de-sangaris_5058096_3210.html>.

164 Dispositions spéciales, supra note 2 au para 31.

165 Ibid au para 13.

166 Ibid au para 24.

167 Stratégie globale, supra note 42 au para 43.

168 Rapport Ban, supra note 22 au para 76. 
La solution la plus adéquate permettant un accès direct aux victimes via l'implication de l'ONU serait alors la mise en place de cours martiales sur le terrain ${ }^{169}$. ONU Femmes a également proposé la création d'un tribunal international qui serait spécifique au traitement des réclamations des victimes d'agressions sexuelles et de viol par des membres des OMPs. Ces projets en sont encore à l'état de discussion pour le moment ${ }^{170}$. En effet, même si ce genre de mesures pourrait effectivement être bénéfique, le chemin est encore long avant qu'elles ne se réalisent étant donné qu'elles impliqueraient non seulement l'accord de l'État hôte de l'OMP, mais également l'accord de chaque État ayant fourni un contingent de soldats puisque ce sont eux qui conservent le privilège de juridiction sur leurs ressortissants. De la même manière, une création d'un tribunal spécial par le Conseil de Sécurité de l'ONU, comme ce fut le cas pour l'ex-Yougoslavie ou le Rwanda, nécessiterait au moins l'accord des cinq membres permanents, ce qui pourrait s'avérer plus compliqué qu'il n'y paraît, les États n'étant pas forcément désireux de voir leurs nationaux jugés par des juridictions autres que les leurs.

Ce serait donc un travail de longue haleine, tant sur le plan juridique que politique, mais pas impossible à réaliser si les Nations Unies décident de s'investir pleinement dans la coopération avec les États contributeurs de troupes afin de relayer les allégations de viols et d'autres agressions sexuelles pour que des enquêtes soient menées de manière approfondie et suffisamment efficace pour permettre l'inculpation des accusés ${ }^{171}$ et pour rendre la justice qui est due aux victimes de ces exactions. Même s'il n'est pas évident pour une organisation internationale de reconnaître sa responsabilité, directe ou indirecte, dans une violation du droit international, il revient à l'ONU de montrer l'exemple aux États à qui elle entend faire respecter ce même droit international. En acceptant de reconnaître sa responsabilité civile et morale, l'ONU n'aurait à y gagner qu'en crédibilité et en légitimité, faisant ainsi taire ses détracteurs, fervents adeptes de l'adage "Who watches the watchers? ».

169 Stratégie globale, supra note 42 au para 59; Dispositions spéciales, supra note 2 au para 59.

170 ONU, Sommet mondial sur l'action humanitaire, «Un nouveau programme d'action pour l'humanité », en ligne : <www.un.org/fr/conf/whs/>; ONU, communiqué, « L'ONU veut renforcer l'égalité des sexes et le leadership des femmes dans les opérations de paix » (13 octobre 2015), en ligne : Centre d'actualités de l'ONU <www.un.org $>$.

171 Deschamps, supra note 157 à la $\mathrm{p} 2$. 